\section{THE INTERNATIONAL EXHIBITION AND CONGRESS OF ELECTRICITY AT PARIS ${ }^{1}$}

II.

THE most crowded place in the Exhibition is the Théatre de l'Opéra. Here from eight to eleven on three evenings in the week are to be seen four long queues waiting for their turn to enter one of the four rooms where the mysterious music is to be heard. Round the walls of each room are hung telephones in pairs, some twenty pairs in all, and the same number of persons are admitted. On putting the telephones to your ears you hear the music which is being performed at the opera-house more than a mile distant. Some of the singers seem to be on your right hand, others on your left, and it sometimes happens that a particular voice is quite piercing in its loudness. There are in fact ten transmitters disposed along the front of the stage, near the footlights, and ten wires leading from them, two of which are connected with the telephones intended for your two ears. Special precautions are taken to prevent the action of the transmitters from being disturbed by the tremors of the boards under the feet of the actors, the transmitters being supported on india rubber and loaded with lead. The telephonic apparatus employed is that of the Ader system.

The greatest novelty as regards principle is exhibited in Dolbear's telephone, in the United States department. The receiver has no magnet, but has two parallel metallic plates near together, and electrically insulated from each other. One of them is connected with the line wire, and the other (in the specimen here exhibited) with the return wire. These two wires are connected with the terminals of the secondary coil of a small Ruhmkorff at the sending station; and the voice of the speaker produces variations in the primary current, on the usual plan of varying the resistance in the circuit of a local battery by variations of pressure. The secondary circuit is not completed, inasmuch as the two plates do not touch; but the opposite electricities which are transmitted to them attract each other on electrostatic principles, and the plates are thus made to vibrate in unison with the voice of the speaker at the sending station. The instrument exhibited is very effective, and reproduces a whisper with greatly increased intensity. It is claimed that this invention does away with the disturbance experienced in other telephones from currents in neighbouring wires, inasmuch as such currents will not affect the attraction between the plates. We should add that the instrument exhibited speaks fairly even when the plate next the ear is disconnected from the wire intended for it, but of course less loudly than when the connection is made. This is just what one would expect from electrostatic attraction, the attraction of a charged for an uncharged body being less than that between two bodies oppositely charged.

We have had an opportunity of seeing the system adopted by Mr. Edison for the measurement of the quantity of electricity consumed in each house which receives a supply from one of his mains. A definite proportion (one thousandth part) of the whole current which goes through the house is shunted through a cell containing two copper plates in a solution of sulphate of copper. The positive plate loses, and the negative plate gains, an amount of copper exactly proportional to the quantity of electricity which passes. There are two such cells in series, one serving as a check upon the other, and the whole arrangement is kept under lock and key, to be opened only by Mr. Edison's agents when they come round to inspect the meters. As the lamps supplied (of a given type) are almost precisely alike in their resistance, and the current, when flowing, is always nearly the same, this arrangement gives a practically accurate measure of the illuminating power supplied.

Much interest has been excited by the exhibition of three magneto-electric machines constructed by Prof. Pacinotti of the University of Cagliari. One of these, constructed at Pisa in 1860 , is the earliest example of the principle of the ring-shaped armature, since embodied in the machines of Gramme and Brush. It was originally constructed as an engine to be driven by a current from without; but it was also used as a generator of electricity, and both these uses of it were described in a paper in the Nuovo Cimento in 1864. The machine contains an iron ring like an anchor ring, round successive portions of which are wound coils of insulated copper wire in depressions cut in the ring to receive them. The intervening portions of the ring are thus (as in the Brush machine) enabled to come very nearly into contact with the surrounding fixed magnets. These consist of two half rings which are the pole pieces of two straight electromagnets. The coils above mentioned are connected in a series, and their junctions are in connection with the several segments of a commutator, as in the Gramme machine.

The second machine was constructed in 1873 , and described in the Nuovo Cimento in 1874 . It is a generator of electricity, of the kind now known as the shunt dynamo, that is to say, the current generated is divided in parallel circuit between the fixed electro-magnet and the external resistance. This is done by means of two pairs of brushes making contact with different sections of the revolving commutator. The ring is replaced by a flat cylinder, across which the successive coils are wound in depressions made for the purpose, the directions of winding being the same as in Siemens' continuous current machine, which was invented about the same time. The connections of the successive coils with one another and with the segments of the commutator are the same as in the first machine.

The third machine, which was constructed in 1878 on a model dating from 1875 , is of a type of which, so far as we know, it is the only example. The idea of it is taken from the well-known experiment (Arago's rotations) in which a revolving horizontal copper disk causes a large magnetised needle balanced above it to revolve in the same direction. The explanation of the effect was first given by Faraday. It depends on the action of a current generated in the copper disk by its motion in the magnetic field due to the needle. The strongest current flows along that diameter which is parallel to the needle, and the current is completed through the circumferential portions of the disk. Pacinotti virtually cuts away all except the diametral portion and one of the two circumferential portions ; in other words, he takes a wire and bends it into the shape of the letter D. This is one convolution of his revolving coil; the next is like the same $D$ tilted a little; the next is tilted a little more, and so on; so that some of the convolutions have the positions-

\section{$\begin{array}{llllllll}D & \square & 0 & 0 & a & 0 & 0 & 0\end{array}$}

the straight part of the wire passing through or nearly through the axis of the coil, and the curved part being in the circumference. There is no room for a core in the ordinary sense, as the wires occupy nearly the whole interior space; but pieces of iron are so disposed partly within and partly without the coil as to serve the purpose of a core, by increasing the induction of the fixed magnets.

(To be continued.) 